\title{
Therapeutic effect of apocynin through antioxidant activity and suppression of apoptosis and inflammation after spinal cord injury
}

\author{
YIJUN SUN, FUTAI GONG, JICHAO YIN, XIAOYAN WANG, XIANGYANG WANG, QING SUN, \\ ZHIQIANG ZHU, XIAOQIANG SU, JIE ZHENG, LI LIU, YANG LI, XINGLV HU and JIA LI \\ Department of TCM Orthopedics and Traumatology, \\ Xi'an City Hospital of Traditional Chinese Medicine, Xi'an, Shaanxi 710021, P.R. China
}

Received November 21, 2015; Accepted November 10, 2016

DOI: $10.3892 /$ etm.2017.4090

\begin{abstract}
Spinal cord injury (SCI) is a devastating condition affecting hundreds of thousands of people worldwide annually. SCI results in activation of the inflammatory response and apoptosis, and generates oxidative stress, which has deleterious effects on the recovery of motor function. Apocynin, an inhibitor of NADPH oxidase, has been demonstrated to improve neuronal functional recovery in rat models of SCI. However, the efficacy of apocynin treatment post-SCI has not been investigated. The aim of this study was to observe the effects of apocynin on the repair of acute spinal cord damage in rats and to examine the potential beneficial effects. A rat model of SCI was established, and apocynin $(50 \mathrm{mg} / \mathrm{kg})$ was administered intraperitoneally at $30 \mathrm{~min}$ after SCI and then every $12 \mathrm{~h}$ for 3 days. In order to examine oxidative tissue injury, the levels of malondialdehyde and glutathione and activities of myeloperoxidase and superoxide dismutase in the spinal cord tissues were measured. Histological evaluations were also conducted. NeuN labeling, TUNEL staining and caspase 3 immunohistochemical staining were performed to analyze neuronal damage and apoptosis around the lesion. Immunohistochemical analysis was also carried out to observe the expression of CD11b and glial fibrillary acidic protein. The expression levels of bax, bcl-2, tumor necrosis- $\alpha$, interleukin (IL)-1 $\beta$ and IL-6 in the spinal cord tissue were assayed by western blotting. Finally, locomotor function was evaluated using the inclined plane test and Basso, Beattie and Bresnahan scores. The results showed that treatment with apocynin decreased oxidative damage, alleviated neuronal apoptosis, inhibited the inflammatory response and resulted in the promotion of locomotor function. Therefore, this study confirmed the therapeutic efficacy of apocynin in the repair
\end{abstract}

Correspondence to: Professor Futai Gong, Department of TCM Orthopedics and Traumatology, Xi'an City Hospital of Traditional Chinese Medicine, 69 Fengcheng 8 Road, Xi'an, Shaanxi 710021, P.R. China

E-mail: gongfutai@163.com

Key words: apocynin, apoptosis, inflammation, oxidative stress, spinal cord injury of SCI, which was probably mediated via the inhibition of apoptosis and the inflammatory response, thus promoting the restoration of nerve function.

\section{Introduction}

Spinal cord injury (SCI), which is attributed to various factors that include mechanical factors as well as other mechanisms caused by the trauma, often leads to the severe dysfunction of limbs and trunk below the damaged section, and is a major cause of permanent disability in children and adults $(1,2)$. In addition to direct injury caused by primary trauma following SCI, a variety of extended neuropathophysiological alterations occur. Secondary damage plays a part in increasing the extent of the pathological effects of SCI where excitotoxicity, inflammation, autophagy as well as apoptosis play vital roles (3-6). Over the past decades various strategies have been used to elucidate the molecular basis of SCI; however, no fully effective treatments for SCI are available to date. Therefore, the development of a safe and efficient treatment for SCI is greatly complicated by the existence of a highly complex injury environment.

Oxidative stress has been implicated to play a significant role in the pathology of SCI $(7,8)$. Nicotinamide adenine dinucleotide phosphate (NADPH)-oxidase is a membrane enzyme composed of several subunits that include NOX and phox subunits, and is responsible for the production of reactive oxygen species (ROS). A previous study has suggested that NADPH oxidase is expressed in neurons, astrocytes and microglia (9). Another study has indicated that NADPH oxidase-derived ROS is involved in the modulation of neurological function under physiological and pathological conditions (10), and inhibition of this enzyme represents an attractive therapeutic target for the treatment of a number of nervous system diseases.

Apocynin, an inhibitor of NADPH oxidase, is a natural organic compound isolated from the roots of Apocynum cannabinum (Canadian hemp) (11). Studies have been conducted to determine its disease-fighting capabilities and application in several types of brain damage, such as traumatic brain injury and stroke $(12,13)$. Notably, the beneficial effects of apocynin following SCI in rats have been demonstrated to be associated with its antioxidant and 
anti-inflammatory properties (14). Therefore, the protective effect and mechanisms of apocynin after SCI require further exploration.

In the present study, the effect of apocynin on oxidative stress, apoptosis, inflammation and function following SCI were examined in rat model. It was found that SCI-induced oxidative damage, neuronal injury, microglial activation and motor deficits were prevented through the anti-apoptotic and anti-inflammatory effects of apocynin. Therefore, the present results suggest that treatment with apocynin following SCI may have the potential to reduce SCI-induced neuronal death.

\section{Materials and methods}

Experiment animals and SCI model. Adult male Sprague-Dawley rats (250-300 g) were obtained from Xi'an Medical University Experimental Animal Center (Xi'an, China). The animals were housed in a temperature- and humidity-controlled environment $\left(22-24^{\circ} \mathrm{C}, 55+5 \%\right.$ humidity and a standard $12 \mathrm{~h}$ light/dark cycle), and supplied with food and water ad libitum. A total of 60 rats were utilized in this study. All experimental protocols were approved by the Xi'an Medical University Animal Care and Use Committee. SCI models were created using standardized mid-thoracic spinal cord compression injury as described by Rivlin and Tator (15), which has been well established in our laboratory for several years. Rats were anesthetized with $10 \%$ chloral hydrate $(3 \mathrm{ml} / \mathrm{kg}$; Bio-Rad Biotechnology, Inc., Shanghai, China), and then positioned on a thermostat-controlled heating pad at $37^{\circ} \mathrm{C}$ in a prone position. Under sterile conditions, a longitudinal incision was made on the midline of the back, exposing the paravertebral muscles and then dissection was conducted to expose the T6/T7 vertebrae. Laminectomy was performed, and the spinal cord was exposed without opening the dura mater. In this model, an aneurysm clip with a calibrated closing force of $24 \mathrm{~g}$ was closed around the mid-thoracic spinal cord for $1 \mathrm{~min}$, which rendered the animals completely paraplegic. The incision was sutured and the rats were placed on heat pads $\left(37^{\circ} \mathrm{C}\right)$ for $2-4 \mathrm{~h}$ to maintain normal body temperature until they were completely awake.

Groups and drug administration. Rats were randomly divided into three groups with 20 rats in each: The sham group, SCI group and SCI + apocynin groups. Animals in the sham group were subjected to the surgical procedure described above, with the exception that the aneurysm clip was not applied. Apocynin (Sigma-Aldrich; Merck Millipore, Darmstadt, Germany) was dissolved in dimethyl sulfoxide (DMSO) and physiological saline $(1: 1 ; 1 \mathrm{mg} / \mathrm{ml})$, and then injected $(50 \mathrm{mg} / \mathrm{kg})$ intraperitoneally $30 \mathrm{~min}$ after SCI and then every $12 \mathrm{~h}$ for 3 days. The sham and SCI groups received equal volumes of DMSO and saline intraperitoneally at the same times daily. A sub-group of 5 rats from each group were sacrificed 3 days following SCI, and the remaining rats were subjected to behavioral testing. Rats were anesthetized with $10 \%$ chloral hydrate $(3 \mathrm{ml} / \mathrm{kg}$; Bio-Rad Biotechnology, Inc.). All rats were sacrificed by exsanguination following anesthesia.

Oxidative stress and antioxidant assays

Measurement of tissue myeloperoxidase (MPO) activity. MPO activity in the spinal cord tissues was determined 3 days after SCI as previously described (16). Tissue samples were obtained and homogenized in $50 \mathrm{mM}$ potassium phosphate buffer (PB) with a $\mathrm{pH}$ of 7.0, and centrifuged for $10 \mathrm{~min}$ at $4,000 \mathrm{x} g$ at $4^{\circ} \mathrm{C}$. The pellets were suspended in $50 \mathrm{mM} \mathrm{PB}$ containing $0.5 \%$ hexadecyltrimethylammonium bromide, then subjected to three freeze and thaw cycles with sonication and centrifuged again $\left(10 \mathrm{~min}\right.$ at $4,000 \mathrm{x} g$ at $\left.4^{\circ} \mathrm{C}\right)$. An aliquot of the supernatant $(0.3 \mathrm{ml})$ was added to $2.3 \mathrm{ml}$ reaction mixture containing $o$-dianisidine, $50 \mathrm{mM} \mathrm{PB}$ and $20 \mathrm{mM} \mathrm{H}_{2} \mathrm{O}_{2}$ solution. The rate of change in absorbance was measured spectrophotometrically at $460 \mathrm{~nm}$. MPO activity was expressed as $\mathrm{U} / \mathrm{g}$ tissue.

Measurement of tissue malondialdehyde (MDA) and glutathione (GSH) levels. Spinal cord tissue samples were homogenized with ice-cold $150 \mathrm{mM} \mathrm{KCl}$ for the determination of MDA and GSH levels. The levels of MDA were assayed for products of lipid peroxidation in the spinal cord tissues homogenates using the thiobarbituric acid (TBA) reaction method, as described previously (17). MDA levels which were determined by measurement of absorption at a wavelength of $532 \mathrm{~nm}$ following reaction with TBA to form a pink chromogen, were expressed as nmol MDA/g tissue. GSH measurements were performed using a kit supplied by Cayman (Cayman Chemical Company, Ann Arbor, MI, USA) according to the manufacturer's instructions. GSH levels were expressed as mmol GSH/g tissue.

Measurement of superoxide dismutase (SOD) activity. SOD activity in the spinal cord tissues was measured with a Shimadzu UV-2100 spectrophotometer (Shimadzu International Trading (Shanghai) Co., Ltd., Shanghai, China). The assay for SOD was based on the activity of this enzyme in the xanthine-xanthine oxidase system (18). The changes in absorbance were measured at $550 \mathrm{~nm}$ with a spectrophotometer. The SOD levels were expressed as activity units per g protein.

Histological analysis. Histological examination of the SCI was performed by NeuN and terminal deoxynucleotidyl-transferase-mediated dUTP nick end labeling (TUNEL) staining as described previously (19). At day 3 after SCI, animals were anesthetized and transcardially perfused with $4 \%$ paraformaldehyde (PB, pH 7.4). A 5-mm spinal cord segment, $2.5 \mathrm{~mm}$ caudal and $2.5 \mathrm{~mm}$ rostral to the injury site, was extracted. Spinal cords were kept in $4 \%$ paraformaldehyde for $24 \mathrm{~h}$ and then transferred to a $30 \%$ sucrose solution (0.1 M PBS, $\mathrm{pH}$ 7.4). Spinal cords were then cut into $30-\mu \mathrm{m}$ axial sections. The sections were permeabilized using $0.4 \%$ Triton X-100 for $30 \mathrm{~min}$, then incubated with $5 \%$ normal donkey serum (Bio-Rad Biotechnology, Inc.) for $1 \mathrm{~h}$ at room temperature, followed by incubation with mouse anti-NeuN monoclonal antibodies (diluted 1:100; ab104224; Abcam, Cambridge, $\mathrm{UK}$ ) overnight at $4^{\circ} \mathrm{C}$. The following day, the sections were incubated with secondary antibodies (Alexa Fluor 488 donkey anti-mouse IgG; diluted 1:100; ab150105; Abcam) at room temperature for $2 \mathrm{~h}$. TUNEL staining was performed using an In Situ Cell Death Detection kit (Roche Diagnostics GmbH, Mannheim, Germany). Sections were incubated with TUNEL reaction mixture including TdT enzyme and TMR red labeled dUTP in a dark humidified chamber for $1 \mathrm{~h}$ at $37^{\circ} \mathrm{C}$, 
followed by three final washes, each for $10 \mathrm{~min}$, and visualized using converter-POD (a horseradish peroxidase-conjugated anti-fluorescein antibody) with $0.03 \%$ 3,3'-diaminobenzidine (DAB). Immunofluorescence was detected and photographed in the dorsal column region within the 5-mm region of interest using a laser scanning confocal microscope (Olympus FluoView ${ }^{\mathrm{TM}}$ FV1000; Olympus Corporation, Tokyo, Japan). For quantitative analysis, the numbers of surviving neurons and TUNEL-positive apoptotic cells were quantified as previously described using pixel density measurements in Scion Image 4.02 (Scion Corporation, Boston, MA, USA) (20). For all stains, at least five sections taken from regular intervals within the 5-mm region of interest were evaluated.

Immunohistochemical analysis. Immunohistochemical staining for spinal cord tissue was performed on ice-cold sections $(30 \mu \mathrm{m})$. Briefly, sections were blocked with $0.01 \mathrm{~mol} / 1$ phosphate-buffered saline (PBS) containing 5\% goat serum for $1 \mathrm{~h}$ at room temperature. Sections were then incubated overnight at $4{ }^{\circ} \mathrm{C}$ with rabbit anti-caspase 3 polyclonal antibodies (ab4051; diluted 1:100), mouse anti-CD11b monoclonal antibodies (ab1211; diluted 1:100) and mouse anti-glial fibrillary acidic protein (GFAP) monoclonal antibodies (ab10062; diluted 1:100), all from Abcam, and then with horseradish peroxidase-conjugated anti-rabbit (ab150077) or mouse (ab6785) IgG antibodies (diluted 1:500), all from Abcam, for $30 \mathrm{~min}$. DAB was used to reveal the immunohistochemical reaction. Primary antibodies were replaced with PBS in the negative control. Cell images were captured with a microscope (Nikon Eclipse E800; Berlin, Germany) equipped with a Spot RT digital camera (Diagnostic Instruments Inc, Sterling Heights, CA, USA). The number of caspase 3-positive cells was counted manually under a high-power field (x400) and the mean percentage of positive cells in six different non-overlapping fields of view (three gray matter and three white matter fields) from each slide was calculated.

Western blot analysis. Rats were euthanized as described above, and the spinal cord was rapidly isolated. Proteins were extracted from the spinal cord $\left(4^{\circ} \mathrm{C}\right)$ using protein extraction reagent (Bio-Rad Biotechnology) surrounding the injured area and the protein concentration was quantified using a BCA protein assay kit (Beijing Solarbio Science \& Technology Co. Ltd., Beijing, China) according to the manufacturer's protocol. Samples were loaded ( $3 \mu 1$ per lane) onto SDS-polyacrylamide gel $[0.8 \% \mathrm{w} / \mathrm{v}$ acrylamide bisacrylamide $(2.5 \mathrm{ml}), 1.0 \mathrm{M}$ Tris-Cl pH $8.8(3 \mathrm{ml}), 20 \%$ SDS $(38 \mu \mathrm{l})$ and $\left.\mathrm{H}_{2} \mathrm{O}(1.9 \mathrm{ml})\right]$ for electrophoresis. Separated proteins on the gel were transferred onto PVDF membranes (Roche Diagnostics). The blots were blocked with $5 \%$ non-fat milk for $2 \mathrm{~h}$ at room temperature, followed by incubation with primary antibodies at $4^{\circ} \mathrm{C}$ overnight. The antibodies comprised rabbit anti-bax polyclonal antibodies (ab32503; diluted 1:1,000), rabbit anti-bcl-2 polyclonal antibodies (ab32124; diluted 1:500), rabbit anti-tumor necrosis factor (TNF)- $\alpha$ polyclonal antibodies (ab6671; diluted $1: 500)$, rabbit anti-interleukin (IL)-1 $\beta$ polyclonal antibodies (ab2105; diluted 1:500), rabbit anti-IL-6 polyclonal antibodies (ab6672; diluted 1:500) and rabbit anti- $\beta$-actin polyclonal antibodies (ab8227; diluted 1:1,000) all from Abcam. The membranes were then incubated with secondary antibodies (goat anti-rabbit IgG; ab6721; Abcam) at $37^{\circ} \mathrm{C}$ for $1 \mathrm{~h}$. The bands were illuminated using an ECL system (Santa Cruz Biotechnology, Inc., Dallas, TX, USA). Immunoreactive bands of all proteins were normalized to the intensity of the corresponding bands for $\beta$-actin. Densitometric analysis of the blots was performed using National Institutes of Health image 1.41 software (Bethesda, MD, USA).

\section{Behavioral assessment}

Inclined plane test. Behavior was evaluated days 1,3,5,7 and 14 postoperatively using the modified Rivlin's method (21), which tested the ability of animals to balance on elevated wooden beams. A simple device was constructed containing a moveable plate with an adjustable angle of $0-90^{\circ}$. The rat's head was placed faced forward, and the angle of inclination between the inclined plane and the horizontal plane was increased gradually, until the rats were unable to maintain a constant position for $5 \mathrm{sec}$. The angle was considered to be the critical value and then recorded.

Basso, Beattie and Bresnahan (BBB) scores. Assessment of motor function recovery of the hind limbs was conducted using the BBB scale, which is based on a 21-point scale originally developed for the SCI rat model (22). Perfectly healthy animals are assigned a locomotor score of 21, and animals with complete hind limb paralysis are scored as 0 . The BBB scores were determined by at least two observers blinded to the treatment. Rats were forced to walk in an open field for $20 \mathrm{~min}$ at days $1,3,5,7$ and 14 postoperatively and their hind limb movement was observed. The average rat hind limb motor function scores on the BBB scale were recorded.

Statistical analysis. All experiments were repeated three times and similar results were obtained. All data are presented as the mean \pm standard deviation and analyzed using SPSS 16.0 software (SPSS, Inc., Chicago, IL, USA). The significance of differences in the experimental results was determined using one-way analysis of variance. $\mathrm{P}<0.05$ was considered to indicate a statistically significant difference.

\section{Results}

Regulatory effect of apocynin on secondary oxidative stress following SCI. SCI caused a significant increase in the MPO activity of spinal cord tissues when compared with the MPO activity of the tissues from sham rats (Fig. 1A; $\mathrm{P}=0.007$ ). In the SCI model rats that received apocynin treatment, the elevations in MPO activity were decreased compared with those in the SCI group $(\mathrm{P}=0.028)$. In the spinal cord tissues of the SCI group, levels of MDA, an index of lipid peroxidation, were found to be significantly higher than those of the sham group (Fig. 1B; $\mathrm{P}=0.005$ ), whereas treatment with apocynin attenuated the SCI-induced elevations in MDA levels $(\mathrm{P}=0.012)$. In accordance with the increased MPO activity and MDA levels, the key antioxidant enzymes GSH and SOD, were found to be significantly depleted in the spinal cord tissues of the SCI group (Fig. 2; $\mathrm{P}=0.003$ ), whereas the antioxidant enzyme activities of the tissues were preserved in the apocynin-treated SCI rats (Fig. 2; $\mathrm{P}=0.026$ ). 

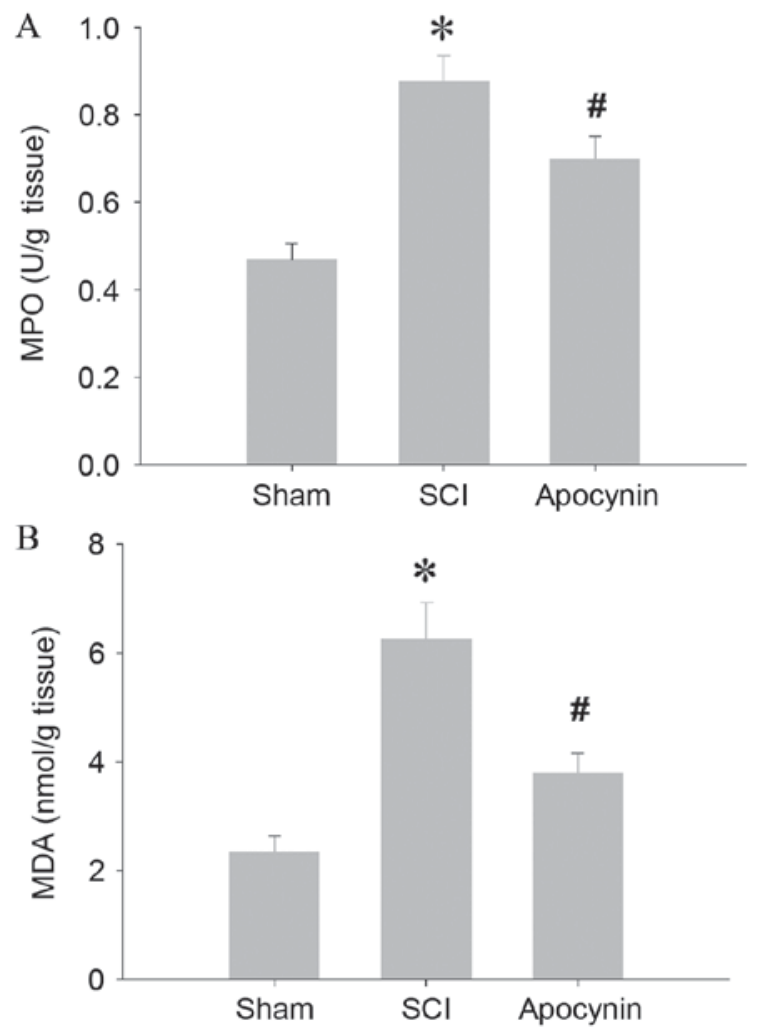

Figure 1. (A) MPO activity and (B) MDA levels in the sham, SCI and apocynin-treated groups. Data are presented as mean \pm standard deviation $(\mathrm{n}=5)$. ${ }^{*} \mathrm{P}<0.01$ vs. the sham group; ${ }^{*} \mathrm{P}<0.05$ vs. the $\mathrm{SCI}$ group. MPO, myeloperoxidase; MDA, malondialdehyde; SCI, spinal cord injury.

Apocynin treatment attenuates neuronal injury in spinal cord tissues following SCI. The effect of apocynin on neuronal injury around the damaged area following SCI was investigated. As shown in Fig. 3, representative photomicrographs and cell counting showed that SCI induced a significant loss of spinal cord neurons, as indicated by a marked reduction of NeuN-positive cells in the SCI group compared with the sham group (Fig. 3B; $\mathrm{P}=0.004$ ). In addition, the number of TUNEL-positive cell was markedly increased in the spinal cord of SCI group, compared with sham (Fig. 3C; $\mathrm{P}=0.001$ ). Notably, apocynin treatment strongly attenuated neuronal cell death in the spinal cord, as indicated by a decreased number of TUNEL-positive cells and increased number of NeuN-positive cells compared with the SCI group (Fig. 3B, P=0.036; Fig. 3C, $\mathrm{P}=0.025$ ). These observations demonstrate that apocynin protects the spinal cord from SCI-induced neuronal cell death.

Apocynin treatment attenuated apoptosis. Neuronal apoptosis was also assessed by the analysis of activated caspase 3 expression using immunohistochemical staining. As shown in Fig. 4A, representative photomicrographs revealed a high density of caspase 3-positive cells in the SCI group compared with sham group (Fig. 4A, $\mathrm{P}=0.002$ ). Following treatment with apocynin for 3 days, the expression of activated caspase 3 in the cells was significantly decreased compared with that in the $\mathrm{SCI}$ group (Fig. 4B, $\mathrm{P}=0.017$ ). To investigate further the mechanisms of apocynin apoptosis blockade, the changes in bcl-2 family members, including the proapoptotic protein bax and antiapoptotic protein bcl-2, in the area of injury were analyzed
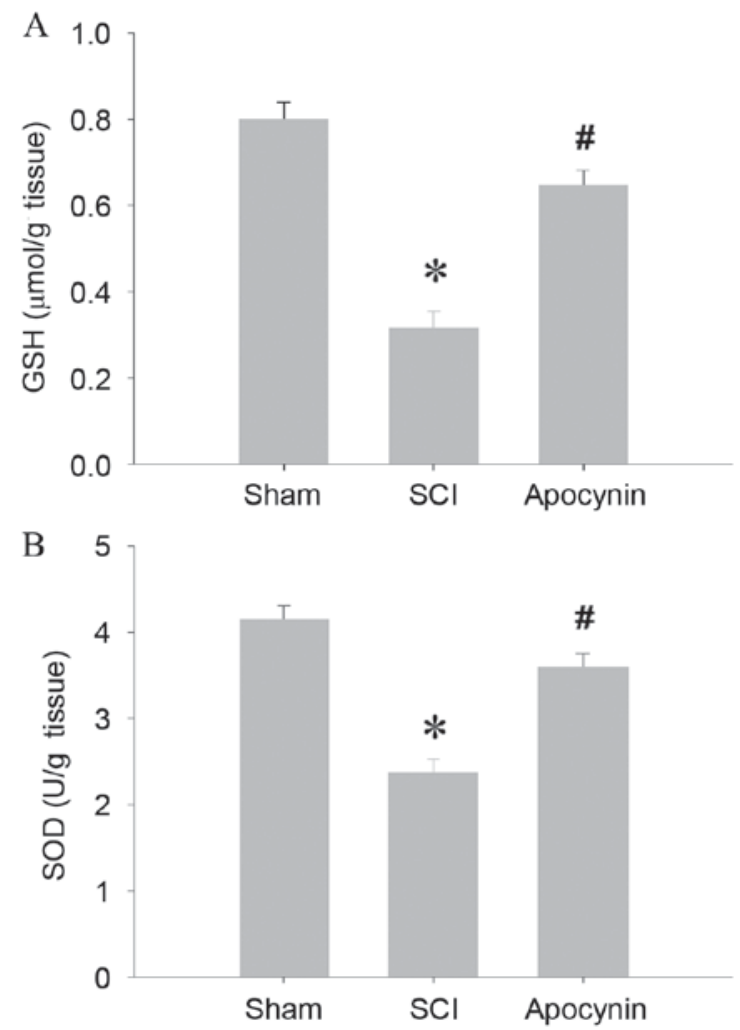

Figure 2. (A) GSH and (B) SOD activity in the spinal cord tissues of the sham, SCI and apocynin-treated groups. Data are presented as mean \pm standard deviation $(\mathrm{n}=5)$. ${ }^{*} \mathrm{P}<0.01$ vs. the sham group; ${ }^{*} \mathrm{P}<0.05$ vs. the SCI group. GSH, glutathione; SOD, superoxide dismutase; SCI, spinal cord injury.

using western blot analysis. The results showed that injury significantly upregulated bax expression $(\mathrm{P}=0.003)$ and downregulated bcl-2 expression $(\mathrm{P}=0.004)$ in the injured spinal cord tissue. Notably, treatment with apocynin significantly decreased the bax protein level $(\mathrm{P}=0.024)$ and increased the bcl-2 level $(\mathrm{P}=0.018)$ compared with that in the SCI group (Fig. 4C and D).

Apocynin treatment reduced inflammatory cytokine levels. To further explore the effect of apocynin, changes in expression levels of CD11b (as a marker for microglia) and GFAP (as a marker for astrocytes) in the spinal cord tissue were tested using immunohistochemical staining. As shown in Fig. 5A, the tissue from SCI model rats clearly had stronger staining for CD11b- and GFAP-positive stains than that from sham rats. However, in the groups treated with apocynin, the expression levels of CD11b and GFAP were markedly less than those in SCI group. In addition, the protein expression levels of TNF- $\alpha$, IL-1 $\beta$ and IL- 6 were detected by western blot analysis. In comparison with the sham group, the protein levels of these markers were increased significantly in SCI group on day 3 post-SCI, and treatment with apocynin significantly downregulated the levels of TNF- $\alpha(\mathrm{P}=0.013), \mathrm{IL}-1 \beta(\mathrm{P}=0.019)$ and IL-6 ( $\mathrm{P}=0.026)$ at the same time point following SCI (Fig. 5B and $\mathrm{C}$ ). These results indicate that apocynin significantly reduces the production of inflammatory factors in the spinal cord tissue in a rat model of SCI.

Protective effects of apocynin on spinal cord motor function in SCI rats. Finally, whether apocynin is able to improve 
A
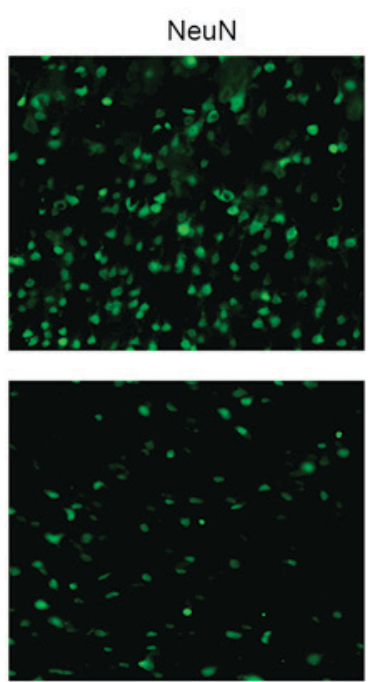

$\mathrm{SCl}$

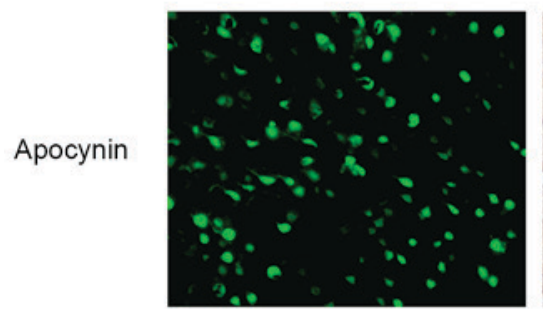

TUNEL

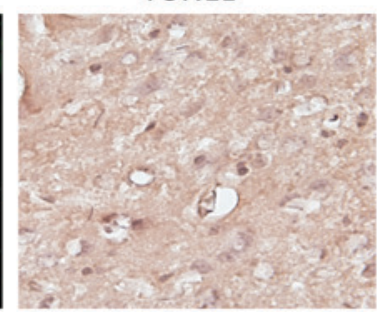

B 70

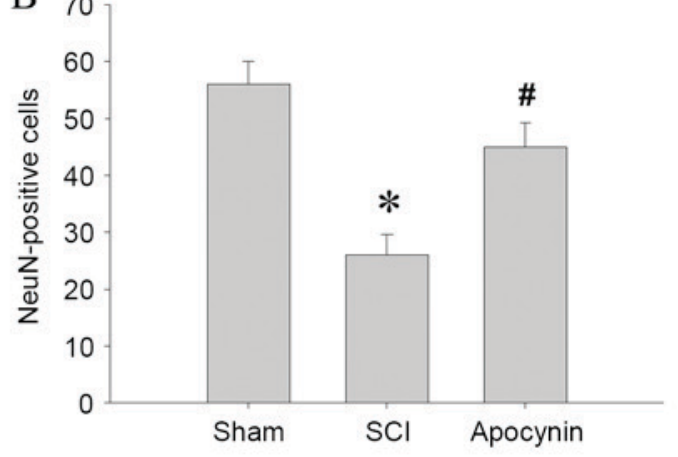

$\mathrm{C}$

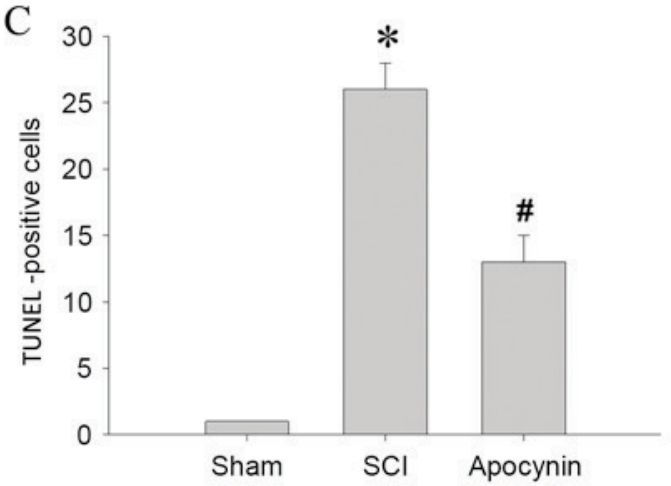

Figure 3. Effects of apocynin on the number of NeuN- and TUNEL-positive cells in the spinal cord tissue at 3 days after SCI. (A) Representative immunofluorescence staining of NeuN and TUNEL in the spinal cords of the sham, SCI and apocynin-treated rats. Alexa Fluor 488 secary antibody was used to detect NeuN (green). Magnification, x100. Quantification of (B) NeuN- and (C) TUNEL-positive cells was performed by counting the number of surviving neurons and apoptotic neurons around the damaged area. Data are presented as mean \pm standard deviation $(n=5)$. NeuN-positive surviving cells showed intact, round nuclei and TUNEL-positive apoptotic cells showed clearly condensed nuclei ${ }^{*} \mathrm{P}<0.01$ vs. the sham group; ${ }^{*} \mathrm{P}<0.05$ vs. the SCI group. SCI, spinal cord injury; TUNEL, terminal deoxynucleotidyl-transferase-mediated dUTP nick end labeling.

the recovery of locomotor function in SCI rats by regulating secondary oxidative stress and suppressing apoptosis and inflammatory response following SCI was evaluated. Locomotion was assessed using the inclined plane test (the modified Rivlin's method) and the BBB locomotor scale at $1,3,5,7$ and 14 days post-SCI. The results showed that animals in the SCI group exhibited significant motor deficits at 1,3,5, 7 and 14 days post-SCI compared with the sham group (Fig. 6; $\mathrm{P}<0.01$ vs. sham group). Injured animals receiving apocynin, however, achieved significantly greater plane inclinations and higher BBB locomotor function scores compared with the SCI group (Fig. $6 ; \mathrm{P}<0.05$ vs. SCI group).

\section{Discussion}

In this study, the neuroprotective role of the NADPH oxidase inhibitor apocynin was evaluated and its ability to reduce neuronal injury and improve motor deficits following SCI in rats were investigated. The results show that apocynin led to significant reductions in neuronal damage and improvements in locomotor recovery following SCI. Recovery was observed at the functional and cellular levels, including greater inclination in the inclined plane test and higher BBB scores, reductions in measures of oxidative stress, and reduced apoptosis and inflammatory response through 3 days post-injury.

In addition to the direct injury caused by primary trauma after SCI, secondary spinal cord damage occurs within min and continues for hours or days, leading to further neurological deterioration (23). Secondary SCI involves numerous complex signal transduction systems, forming a large gene regulation network. With greater understanding of SCI, pathophysiological mechanisms and signaling pathways underlying secondary injuries of the spinal cord have been proposed (24). In particular, oxidative stress has been reported to be one of the earliest biochemical changes after SCI, and an important factor contributing to deterioration of the primary injury, which plays a key role in secondary damage following SCI (25). Therefore, it is important to effectively regulate secondary oxidative stress following SCI. A number of studies have focused on the oxidative properties of apocynin. Apocynin has been shown to reduce oxidative stress in the central nervous system during conditions including sepsis (26) and brain injury $(27,28)$, providing significant neuroprotection. Myeloperoxidase (MPO) is a cationic protein present in primary azurophilic granules of neutrophils and monocytes, MDA is the end product of lipid peroxidation, and GSH and SOD are important scavenger enzymes of free radicals. The present study determined their activity in the spinal cord tissues to evaluate oxidative stress injury secondary to SCI. Results from the present study showed that from 3 days post-SCI, spinal cord MPO activity and MDA levels significantly increased, whereas GSH and SOD activity significantly decreased. However, apocynin significantly inhibited the increases in MPO activity and MDA levels, and increased GSH and SOD 

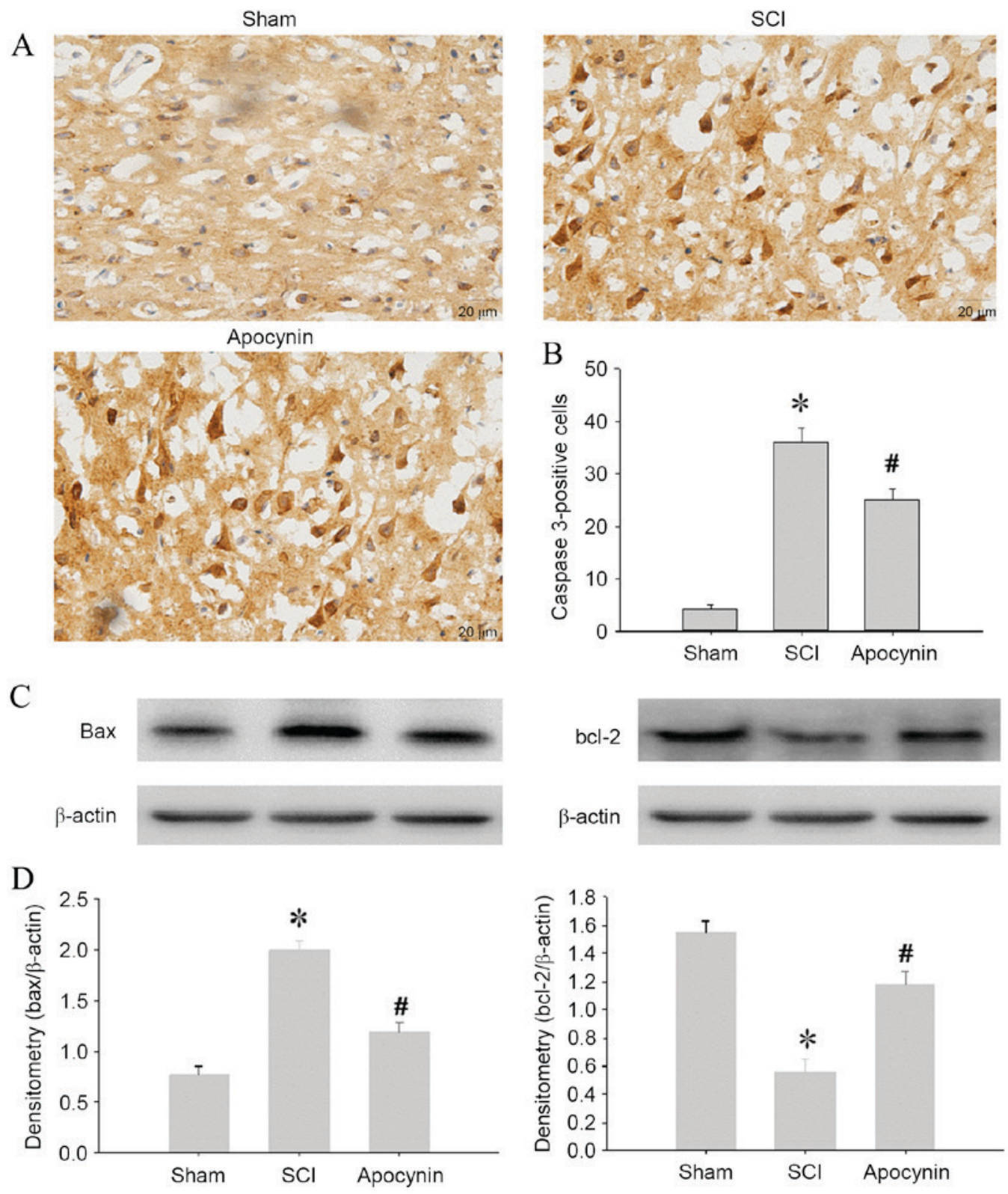

Figure 4. Anti-apoptotic effect of apocynin. (A) Representative immunohistochemical staining of caspase 3 in the spinal cord from sham, SCI and apocynin-treated rats. Scale bar, $20 \mu \mathrm{m}$. (B) Quantification was performed by counting the caspase 3-positive cells in the injured spinal cord from each group. Data are presented as mean \pm standard deviation $(n=5)$. (C) Protein expression of bax and bcl-2 was assessed by western blotting at day 3 in different experimental groups following SCI or sham surgery. (D) Densitometric analysis of bax and bcl-2 bands in comparison with $\beta$-actin. Data are presented as mean \pm standard deviation ( $\mathrm{n}=5)$. ${ }^{*} \mathrm{P}<0.01$ vs. the sham group; ${ }^{\text {P }}<0.05$ vs. the SCI group. SCI, spinal cord injury.

activity post-SCI. These results are similar to those of previous studies reporting that apocynin treatment significantly reduces oxidative stress damage after SCI $(14,26)$.

During the secondary injury following SCI, continuous ischemia and hypoxia induce the release of pro-apoptotic factors and pro-inflammatory cytokines, which mainly mediate activation of MAPK pathways (24). Microglia, which are recruited to the injury site, magnify the extent of inflammation through the secretion of proinflammatory cytokines, including TNF- $\alpha$, IL-1 $\beta$ and IL-6 (29). At the early stage of SCI, reactive astrocytes start to synthesize abundant GFAP and release various cytokines such as nerve growth factor and basic fibroblast growth factor, which promote the recovery of neuron damage (30). However, the level of GFAP, as a marker of astrocytes, reflects the degree of neurological damage. Therefore, prevention of an inflammatory response may be important in neurological recovery. In the present study, immunohistochemical analysis revealed that CD11b and GFAP expression was markedly reduced by apocynin, suggesting that apocynin may contribute to neuronal recovery through attenuating the secretion of inflammatory cytokines. The protein expression levels of TNF- $\alpha$, IL- $1 \beta$ and IL- 6 were detected by western blot analysis. The levels of these proinflammatory cytokines were found to be increased after SCI, demonstrating a systemic inflammatory response to spinal cord trauma, whereas apocynin treatment attenuated these increases. Previous studies have demonstrated a significant increase in the production of these proinflammatory cytokines in an experimental model of $\mathrm{SCI}$ in mice (31), which are consistent with the finding in the present study that the expression levels of TNF- $\alpha$, IL-1 $1 \beta$ and 
A
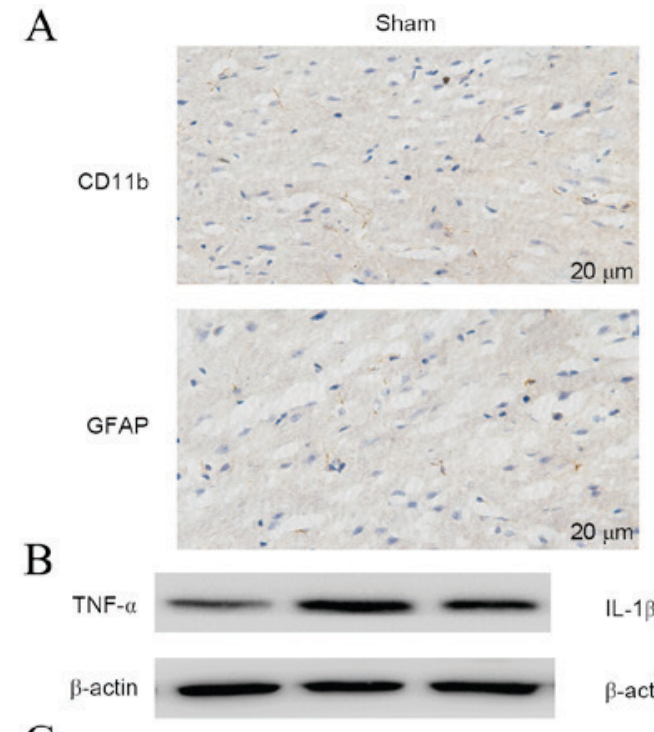

C

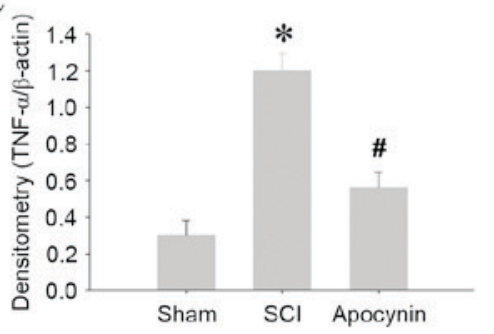

$\mathrm{SCl}$
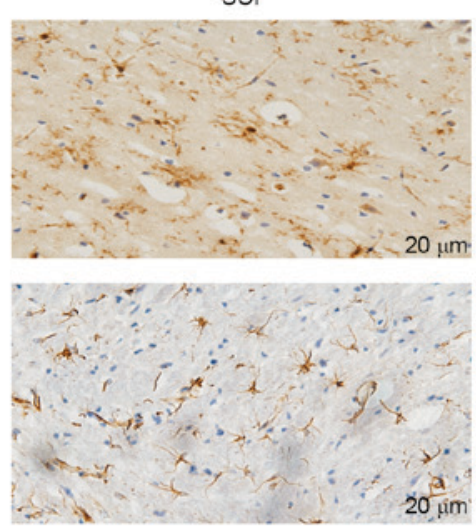

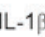

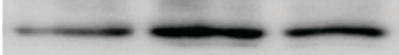

$\beta$-actin
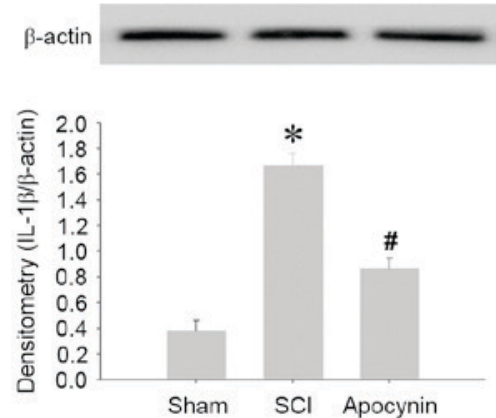
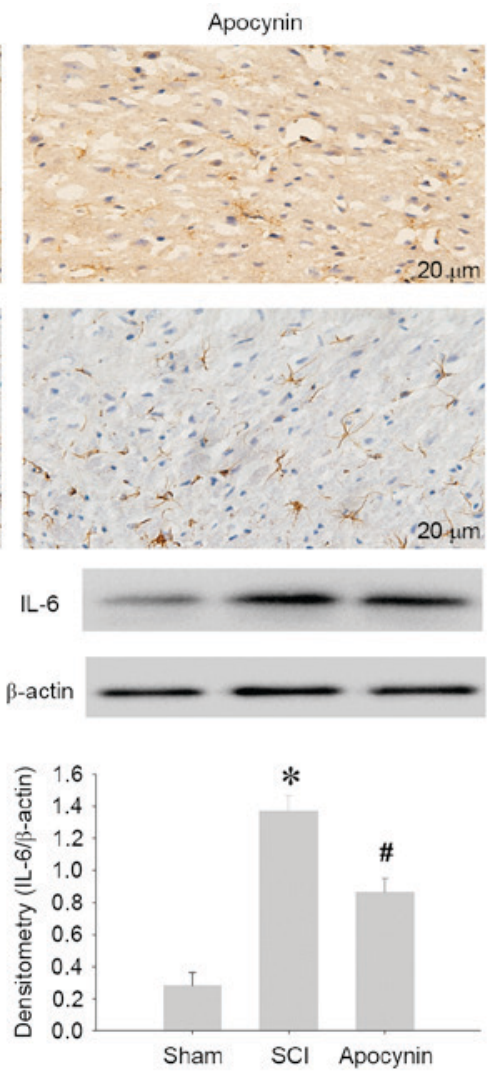

Figure 5. Anti-inflammatory effect of apocynin. (A) CD11b and GFAP expression in the lesion of the spinal cord tissue detected by immunohistochemical staining at 3 days after SCI. (B) Western blots showing the levels of TNF- $\alpha$, IL- $1 \beta$, IL- 6 and $\beta$-actin in the injured spinal cord at 3 days following SCI or sham surgery. (C) Densitometric analysis of TNF- $\alpha$, IL-1 $\beta$ and IL- 6 bands in comparison with $\beta$-actin. Results demonstrated that TNF- $\alpha$, IL- $1 \beta$ and IL- 6 protein expression levels were significantly elevated after SCI. Administration of apocynin significantly decreased the levels of TNF- $\alpha$, IL-1 $\beta$ and IL- 6 protein expression Data are expressed as the mean $\pm \mathrm{SD}(\mathrm{n}=5)$. ${ }^{*} \mathrm{P}<0.01$ vs. the sham group; ${ }^{\#} \mathrm{P}<0.05$ vs. the SCI group. SCI, spinal cord injury; GFAP, glial fibrillary acidic protein; TNF, tumor necrosis factor; IL, interleukin.
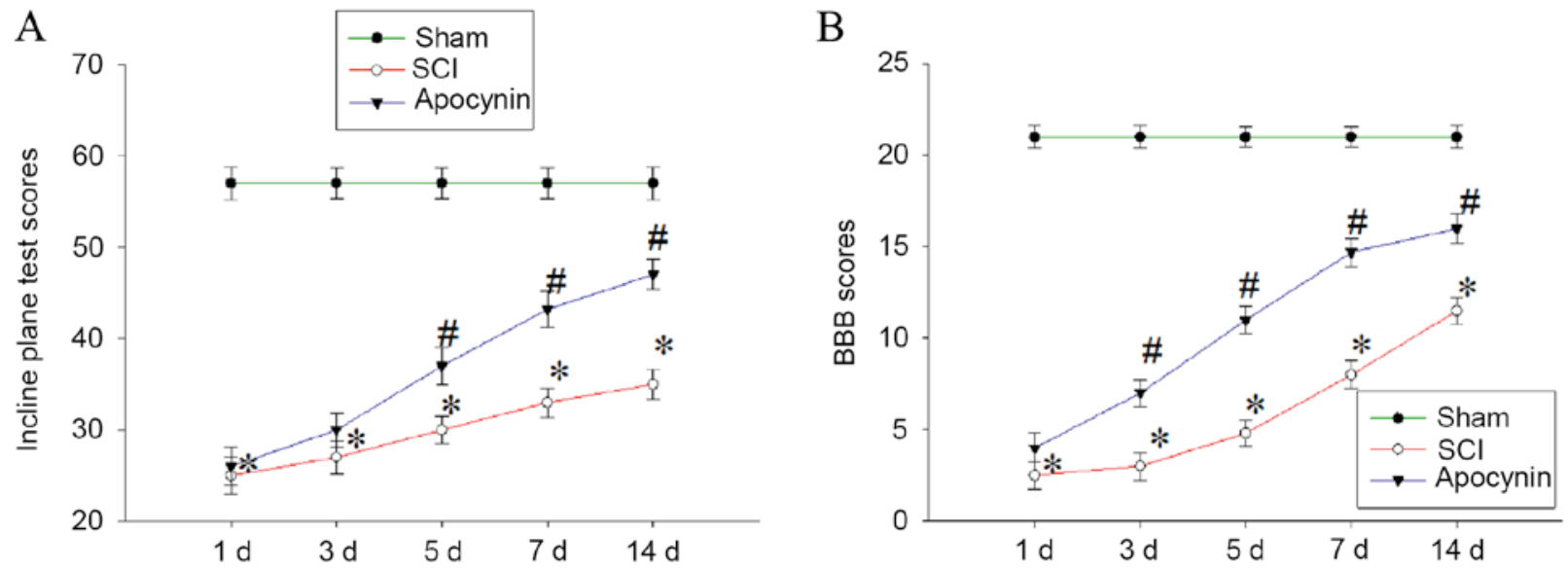

Figure 6. Apocynin promotes functional recovery of the spinal cord in SCI rats. (A) Inclined plane test scores and (B) BBB scores of rats at days $1,3,5,7$ and 14 after SCI or sham surgery. Angle of inclination and the BBB mean score reflect the locomotor function of rats' limbs. Data are presented as mean \pm standard deviation ( $\mathrm{n}=5)$. ${ }^{*} \mathrm{P}<0.01$ vs. the sham group; ${ }^{\text {P }}<0.05$ vs. the SCI group. SCI, spinal cord injury; BBB, Basso, Beattie and Bresnahan; $d$, day.

IL-6 protein were markedly downregulated in rats treated with $50 \mathrm{mg} / \mathrm{kg}$ apocynin for 3 days as compared with those in rats in SCI group, thus limiting neuroinflammation and promoting neurological recovery.

In addition to oxidative stress and inflammatory response, apoptosis is an important mediator of secondary damage after
SCI (32). It initially occurs $6 \mathrm{~h}$ post-injury at the lesion center and continues for several days, with a steadily increasing number of apoptotic cells in this region. Caspase 3, which is an important component of caspase cascades, has been associated with apoptosis in SCI (33). The results of the present study demonstrate apocynin attenuates the degree of apoptosis, measured 
by TUNEL detection and caspase-3 immunohistochemical staining, in the spinal cord following SCI. The numbers of TUNEL and activated caspase 3-positive cells were reduced in apocynin-treated SCI rats compared with those in the SCI group, indicating that apocynin promoted neural functional recovery in SCI rats by decreasing the number of apoptotic cells in the injured spinal cord tissue. Subsequently, the protein expression of bax and bcl- 2 in the injured area were detected by western blot analysis. Bax is a key component in the induction of cellular apoptosis through mitochondrial stress, whereas bcl-2 inhibits mitochondrial permeabilization by binding membrane-inserted bax monomers, thus preventing the functional oligomerization of bax (34). The results of the present study indicate that apocynin significantly increased the expression of the antiapoptotic protein bcl- 2 and decreased the expression of the proapoptotic protein bax in the injured spinal cord tissue compared with the SCI control. These results are consistent with a previous study, which reported that apocynin treatment could significantly suppressed apoptosis after SCI (14).

Finally, whether apocynin was able to improve spinal cord function in SCI rats by regulating secondary oxidative stress and inhibiting apoptosis and inflammation following SCI was investigated. The inclined plane test and 21-point BBB open field locomotor score are widely accepted for assessing locomotor recovery of animals. In this study, following treatment with $50 \mathrm{mg} / \mathrm{kg}$ apocynin every $12 \mathrm{~h}$ for 3 days, the mean values in the inclined plane test and BBB scores were significantly increased compared with those in the SCI group. Following the successful establishment of the SCI model, apocynin promoted the recovery of locomotor function in rats following SCI, and exhibited neuroprotective effects on the spinal cord.

In conclusion, the results of the present study demonstrate that the protective mechanism associated with apocynin against acute SCI may partly depend on anti-apoptotic and anti-inflammatory signaling pathways, downregulation of MPO and MDA, and upregulation of GSH, SOD activity, which together inhibit secondary oxidation following SCI. However, it is unclear whether or not the current level and route of administration of apocynin provides the maximal neuroprotective benefits, and further detailed investigations into the underlying mechanisms are required.

\section{References}

1. Grigorean VT, Sandu AM, Popescu M, Iacobini MA, Stoian R, Neascu C, Strambu V and Popa F: Cardiac dysfunctions following spinal cord injury. J Med Life 2: 133-145, 2009.

2. Kwon BK, Tetzlaff W, Grauer JN, Beiner J and Vaccaro AR: Pathophysiology and pharmacologic treatment of acute spinal cord injury. Spine J 4: 451-464, 2004.

3. Liu D, Xu GY,Pan E and McAdoo DJ: Neurotoxicity of glutamate at the concentration released upon spinal cord injury. Neuroscience 93: 1383-1389, 1999.

4. Hausmann ON: Post-traumatic inflammation following spinal cord injury. Spinal Cord 41: 369-378, 2003.

5. Walker CL, Walker MJ, Liu NK, Risberg EC, Gao X, Chen J and Xu XM: Systemic bisperoxovanadium activates Akt/mTOR, reduces autophagy and enhances recovery following cervical spinal cord injury. PLoS One 7: e30012, 2012.

6. Lu J, Ashwell KW and Waite P: Advances in secondary spinal cord injury: Role of apoptosis. Spine (Phila Pa 1976) 25 1859-1866, 2000.

7. Xiong Y, Rabchevsky AG and Hall ED: Role of peroxynitrite in secondary oxidative damage after spinal cord injury. J Neurochem 100: 639-649, 2007.
8. Khayrullina G, Bermudez S and Byrnes KR: Inhibition of NOX2 reduces locomotor impairment, inflammation, and oxidative stress after spinal cord injury. J Neuroinflammation 12: 172, 2015.

9. Bedard K and Krause KH: The NOX family of ROS-generating NADPH oxidases: Physiology and pathophysiology. Physiol Rev 87: 245-313, 2007.

10. Lo W, Bravo T, Jadhav V, Titova E, Zhang JH and Tang J: NADPH oxidase inhibition improves neurological outcomes in surgically-induced brain injury. Neurosci Lett 414: 228-232, 2007.

11. White CN, Figtree GA, Liu CC, Garcia A, Hamilton EJ, Chia KK and Rasmussen HH: Angiotensin II inhibits the Na+-K+ pump via PKC-dependent activation of NADPH oxidase. Am J Physiol Cell Physiol 296: C693-C700, 2009.

12. Choi BY, Jang BG, Kim JH, Lee BE, Sohn M, Song HK and Suh SW: Prevention of traumatic brain injury-induced neuronal death by inhibition of NADPH oxidase activation. Brain Res 1481: 49-58, 2012.

13. Chen H, Song YS and Chan PH: Inhibition of NADPH oxidase is neuroprotective after ischemia-reperfusion. J Cereb Blood Flow Metab 7: 1262-1272, 2009.

14. Impellizzeri D, Mazzon E, Esposito E, Paterniti I, Bramanti P and Cuzzocrea S: Effect of Apocynin, an inhibitor of NADPH oxidase, in the inflammatory process induced by an experimental model of spinal cord injury. Free Radic Res 45: 221-236, 2011.

15. Rivlin A and Tator $\mathrm{CH}$ : Effect of duration of acute spinal cord compression in a new acute injury model in the rat. Surg Neurol 10: 38-43, 1978

16. Hillegass LM, Griswold DE, Brickson B and AlbrightsonWinslow C: Assessment of myeloperoxidase activity in whole rat kidney. J Pharmacol Methods 24: 285-295, 1990.

17. Beuge JA and Aust SD: Microsomal lipid peroxidation. Methods Enzymol 52: 302-310, 1978.

18. Oyanagui Y: Revaluation of assay methods and establishment of kit for superoxide dismutase activity. Anal Biochem 142: 290-296, 1984 .

19. Zhou C, Tu J, Zhang Q, Lu D, Zhu Y, Zhang W, Yang F, Brann DW and Wang R: Delayed ischemic postconditioning protects hippocampal CA1 neurons by preserving mitochondrial integrity via Akt/GSK3 $\beta$ signaling. Neurochem Int 59: 749-758, 2011.

20. Donnelly DJ, Gensel JC, Ankeny DP, van Rooijen N and Popovich PG: An efficient and reproducible method for quantifying macrophages in different experimental models of central nervous system pathology. J Neurosci Methods 181: 36-44, 2009.

21. Rivlin AS and Tator $\mathrm{CH}$ : Objective clinical assessment of motor function after experimental spinal cord injury in the rat. J Neurosurg 47: 577-581, 1977.

22. Basso DM, Beattie MS and Bresnahan JC: A sensitive and reliable locomotor rating scale for open field testing in rats. J Neurotrauma 12: 1-21, 1995.

23. Blight AR: Morphometric analysis of blood vessels in chronic experimental spinal cord injury: Hypervascularity and recovery of function. J Neurol Sci 2: 158-174, 1991.

24. Song Y, Liu J, Zhang F, Zhang J, Shi T and Zeng Z: Antioxidant effect of quercetin against acute spinal cord injury in rats and its correlation with the p38MAPK/iNOS signaling pathway. Life Sci 92: 1215-1221, 2013

25. Juurlink BH and Paterson PG: Review of oxidative stress in brain and spinal cord injury: Suggestions for pharmacological and nutritional management strategies. J Spinal Cord Med 21: 309-334, 1998.

26. Hernandes MS, D'Avila JC, Trevelin SC, Reis PA, Kinjo ER, Lopes LR, Castro-Faria-Neto HC, Cunha FQ, Britto LR and Bozza FA: The role of Nox2-derived ROS in the development of cognitive impairment after sepsis. J Neuroinflammation 11: $36,2014$.

27. Keirstead HS, Nistor G, Bernal G, Totoiu M, Cloutier F, Sharp K and Steward O: Human embryonic stem cell-derived oligodendrocyte progenitor cell transplants remyelinate and restore locomotion after spinal cord injury. J Neurosci 19: 4694-4705, 2005.

28. Zhang Z, Huang Z, Dai H, Wei L, Sun S and Gao F: Comparison of methylprednisolone and calpain inhibitor for the protection of ischemiareperfusion spinal cord injury in rats. Ortho J Chin 19: 1026-1029, 2011

29. Fehlings MG and Nguyen DH: Immunoglobulin G: A potential treatment to attenuate neuroinflammation following spinal cord injury. J Clin Immunol 30 (Suppl 1): S109-S112, 2010. 
30. Faulkner JR, Herrmann JE, Woo MJ, Tansey KE, Doan NB and Sofroniew MV: Reactive astrocytes protect tissue and preserve function after spinal cord injury. J Neurosci 24: 2143-2155, 2004

31. Impellizzeri D, Esposito E, Mazzon E, Paterniti I, Di Paola R, Bramanti P, Morittu VM, Procopio A, Perri E, Britti D and Cuzzocrea S: The effects of a polyphenol present in olive oil, oleuropein aglycone, in an experimental model of spinal cord injury in mice. Biochem Pharmacol 83: 1413-1426, 2012.

32. Rong W, Wang J, Liu X, Jiang L, Wei F, Hu X, Han X and Liu Z: Naringin treatment improves functional recovery by increasing BDNF and VEGF expression, inhibiting neuronal apoptosis after spinal cord injury. Neurochem Res 37: 1615-1623, 2012.
33. Wei H, Teng H, Huan W, Zhang S, Fu H, Chen F, Wang J, Wu C and Zhao J: An upregulation of SENP3 after spinal cord injury: Implications for neuronal apoptosis. Neurochem Res 37: 2758-2766, 2012.

34. Dlugosz PJ, Billen LP, Annis MG, Zhu W, Zhang Z, Lin J, Leber B and Andrews DW: Bcl-2 changes conformation to inhibit Bax oligomerization. EMBO J 25: 2287-2296, 2006. 